\title{
Bacterial exchange via nanotubes: lessons learned from the history of molecular biology
}

\author{
Thomas A. Ficht* \\ Department of Veterinary Pathobiology, College of Veterinary Medicine, Texas A\&M University, College Station, TX, USA
}

Edited by:

John R. Battista, Louisiana State

University and A \& M College, USA

\section{Reviewed by:}

Marina Kalyuzhnaya, University of Washington, USA

Garry Myers, University of Maryland USA

\section{*Correspondence:}

Thomas A. Ficht, Department of Veterinary Pathobiology, College of Veterinary Medicine, Texas A\&M University, TAMUs 4467, College Station, TX 77843, USA.

e-mail: tficht@cvm.tamu.edu
DNA transfer between bacteria has a long and storied history. Starting shortly after the discovery by Avery, MacLeod, and McCarty that DNA was the genetic material, the exchange of DNA between bacteria confirmed that DNA transfer could stably change the phenotypic behavior of organisms. Continued effort along these lines led to the discovery of conjugation systems, bacteriophage transduction, bacterial genome mapping, and to some represents the birth of molecular biology. Recent findings by Dubey and Ben-Yehuda (2011) expand on these early results by suggesting that exchange between bacteria may occur continuously under certain growth conditions via nanotubes. These nanotubes have a structure similar to cell membranes and are sensitive to mild detergent treatment. Transfer of protein and plasmid DNA was demonstrated directly between neighboring and distant bacteria of the same and different genera. Transfer of RNA cannot be ruled out and the transfer of chromosomal DNA was not addressed. This work may reveal an important mechanism behind the spread of antibiotic resistance, however, much work remains to be done in order to confirm or refute the role of this mechanism in the dangerous spread of antibiotic resistance within the prokaryotic biosphere. The work of early molecular biology pioneers can be used as inspiration, if not as a direct template to guide future experimental confirmation.

Keywords: nanotubes, DNA transfer, antibiotic resistance, conjugation, adaptation

\section{INTRODUCTION}

Recent studies by Dubey and Ben-Yehuda (2011) reported in the journal Cell indicate that intracellular molecules including metabolites, protein, mRNA, and plasmid DNA may be readily transferred between adjacent bacteria via nanotubes (Dubey and Ben-Yehuda, 2011). The phenomenon is reportedly restricted to cells grown on solid surfaces and specifically induced under this growth condition. Transfer of cellular contents was visualized using green fluorescent protein, GFP to monitor the gradual appearance of fluorescence in cells that did not carry the gene. The presence of nanotubes was visually confirmed by scanning electron microscopy and revealed projections distributed in a non-specific manner on the cell surface of both laboratory and undomesticated strains of Bacillus subtilis. High-magnification analysis of a typical tube revealed a structure comprised of outer and inner layers that hinted at a multilayered membrane structure typical of bacterial membranes. Transfer was observed most rapidly between neighboring cells, but also in cells distal from GFP-positive cells consistent with observations of numerous connections between neighboring cells including a reduced number of elongated connections.

Active transport was visualized within nanotubes connecting neighboring cells using gold particles directed at GFP to corroborate an intercellular path for molecular exchange. In order to establish that transfer did not involve acquisition of material released into the extracellular medium, transfer of calcein was evaluated. Once taken up by the cell, endogenous esterases convert calcein into a fluorescent hydrophilic product $(623 \mathrm{Da})$ that is unable to traverse membranes and is considered trapped within the cytoplasm (Tiberghien and Loor, 1996). Additionally, if released from the cell following autolysis, the esterified product is unable to cross the cellular membrane. Consistent with prediction, this fluorescent molecule was observed to accumulate over time in recipient cells. Although most of their efforts demonstrated transfer between organisms in close contact, the authors point out that " . . as time progresses, GFP-negative cells not directly contacting GFP-positive cells, also gained a fluorescence signal...”. This appears to suggest a possibility for temporary or transient contacts occurring between bacteria or more likely given their growth on solid surfaces that there is interaction over long distances. Exchange was also shown to occur in both directions; i.e., a two-way exchange, as revealed by the ability of both strains to transiently express dual antibiotic resistance. Of note, was the additional possibility that transcripts are also being traded among the cells. Finally, and most importantly, the creation of nanotubes and the transfer of intracellular protein and DNA was demonstrated to occur between unrelated genera suggesting a potential basis for the broad distribution of antibiotic resistance.

\section{ANALYSIS}

Intercellular nanotubes bridge adjacent cells and generate a network of tubular conduits enabling the exchange of cytoplasmic content. The speed of transfer is suggested to inversely correlate with the size of the molecule based on comparison of calcein and GFP transport, as well as, the length and the diameter of the nanotubes that join both neighboring and distal cells. The composition of the nanotubes is suspected to be similar to bacterial membranes based on their general appearance in TEM 
and demonstrated sensitivity to treatment with low concentration SDS. Transfer appears to progress as a normal part of growth and replication of the organism linking the cytoplasmic contents of multiple cells containing proteins, RNAs and metabolites. In contrast to more specialized systems, nanotubes appear to serve as simple conduits allowing the cytoplasmic contents of cells to mix. Although basic in design, the absence of any apparent specific function and their ubiquitous presence could have vast implications with regard to the acquisition or sharing of hereditary traits. Although the experiments described in the previous paragraph document a surprising level of exchange of intracellular contents via nanotubes, of greater potential impact is the transfer of antibiotic resistance. The critical nature of these findings brings to mind early experiments in bacterial genetics and in the birth of molecular biology.

Upon first glance, the current experiments appear to lack the scientific rigor associated with early experiments used to define the exchange of DNAs referred to as mating, known now as conjugation. However, after more careful consideration one should realize that the apparent shortcoming of the current experimentation reflects their relatively early stage of development. Over time, the scientific method will demand that additional experiments be performed and the mechanism behind this phenomenon explained, and the validity of the claims made and the findings documented will be readily acknowledged or forgotten. Yet, the potential for exchange of hereditary material is so broad, and the potential promise for future prevention of infectious disease so great, that comparison with the earlier work is only natural.

In making such comparisons it must be remembered that the pioneering experiments of Joshua Lederberg documenting exchange of bacterial DNA came on the heels of seminal experiments of Avery et al. documenting transformation of " $R$ " pneumococci to virulent " $\mathrm{S}$ " form to prove that genes were composed of DNA (Avery et al., 1944). In 1945 Lederberg proposed that mixing auxotrophs at elevated concentrations would result in the isolation of billions of prototrophic bacteria (Lederberg, 1987). Experiments performed by Beadle and Tatum had previously identified nutritional mutants in Neurospora. However, genetic studies in bacteria were hampered by adherence to classical genetic techniques that relied upon meiosis and the fusion of gametes. Fortunately, despite these limitations, Lederberg prevailed upon Tatum to obtain mutants in Escherichia coli K12 defective in more than one nutritional requirement (Brock, 1990). These mutants proved to be invaluable in the discovery of bacterial genetics and arguably the history of molecular biology. Similar to the experiments described by Dubey and Ben-Yehuda (2011) elevated concentrations of cells on solid media were thought to be necessary to ensure detection of infrequent events. In initial experiments using auxotrophic mutants containing single defects transfer between related or unrelated bacterial strains was undetectable. Based on earlier experiments using single genetic defects, Lederberg recognized spontaneous reversion rates provided a high enough background of prototroph isolation to prevent observation of a significant number of possible matings. In order to enhance the sensitivity of this approach, double mutants would greatly reduce spontaneous reversion to prototrophy and would therefore provide greater sensitivity for detecting the exchange of DNA. Successful demonstration of transfer came with the growth of bacteria on solid media after mixing two genotypes thr ${ }^{-} \mathrm{leu}^{-}$and bio $^{-}$met $^{-}$thi ${ }^{-}$(Lederberg and Tatum, 1946). Not only did the results indicate the exchange of DNA, "mating" between bacteria, the frequency of appearance confirmed the basis for the failure of previous attempts using single mutations, i.e., the rate of reversion was nearly identical to the spontaneous rate of appearance of prototrophs. Of course questions remained. Could autolysis have resulted in the availability of DNA to be taken up by the recipient cells? To disprove this possibility, filtrates were used to demonstrate that this was not a source of transformation (Lederberg, 1947; Tatum and Lederberg, 1947). However, not completely satisfied with this experiment, Avery suggested, and Lederberg later confirmed that the use of DNase treatment confirmed that the release of DNA did not play a role (Brock, 1990). Additional considerations including the secretion of metabolic intermediates to support growth or cross-feeding were progressively eliminated by (i) the use of a U-tube to demonstrate that physical contact was necessary for this phenomenon to occur, and (ii) meticulously purifying and repurifying the prototrophic recombinants including the use of UV irradiation to reduce the number of viable cells within colonies to a singular viable prototroph. Thanks to the work of Hayes we now know that donor strains contain a fertility factor or conjugative plasmid and that donor or $\mathrm{F}^{+}$cells promote the unidirectional transfer of hereditary rather than non-hereditary material into recipient or $\mathrm{F}^{-}$ cells (Hayes, 1952a,b). Furthermore, integration of this conjugative plasmid into the bacterial genome promotes transfer of the entire chromosome into the recipient cells and was used to map the E. coli genome.

In the experiments performed by Dubey and Ben-Yehuda (2011), transfer of non-hereditary phenotypes was first demonstrated using GFP and calcein fluorescence. These experiments documented the appearance of increased transfer over time in a manner that suggested an inverse relationship between accumulation of fluorescence and the distance between donor and recipient cells. Confirmation of direct transfer was revealed by the appearance of fluorescent calcein within recipient cells. Certainly a result that is consistent with transfer via nanotubes, but in order to improve acceptance of these results, the authors should be urged to consider the use of better controls that eliminate the potential for carryover of extracellular calcein or to prove that metabolically active cells are essential to transfer. Consider the possibility that extracellular calcein adhering to the cells could be carried over despite extensive washing causing the appearance of fluorescence in the recipients cells. Although the cells were washed extensively following incubation in calcein, there was no control provided that would account for potential carry over of calcein that may explain the appearance of fluorescence in the recipient bacteria. Additional controls for these experiments may include steps to demonstrate the absence of retention of active calcein on the outer cell surface and determine whether nanotubes are only associated with metabolically active bacteria.

The demonstrated transfer of fluorescent protein, suggested the potential to exchange proteins at a level that could potentially alter bacterial phenotype. In this case, the authors missed 
an opportunity to pay homage to earlier investigations by demonstrating the rescue of auxotrophs similar to the experiments of Lederberg, but perhaps more importantly failed to explore any potential link between the presence of nanotubes and the phenomenon known as bacterial adaptation. Presumably, the ready exchange of intracellular components would allow for greater variation within a colony/culture enhancing the flexibility of an organism to face future challenges. Despite these failings the researchers chose instead to evaluate an issue of serious current concern, i.e., the transfer of antibiotic resistance. Interestingly, the results revealed bidirectional transfer of non-hereditary antibiotic resistance. The frequency of transfer appears to be in the order of 1:700 cells based on the recovery of the $\mathrm{Cm}^{\mathrm{R}}$ cells when selected for nonhereditary resistance to kanamycin. This frequency is much greater than the frequency of conjugation $10^{-6}$ to $10^{-7}$ or plasmid transfer as documented by Dubey and Ben-Yehuda, 2011; below) and appears to rule out spontaneous appearance of antibiotic resistant mutants. Perhaps more importantly, these experiments provide the groundwork for experiments concerning a more serious concern, i.e., hereditary transfer of antibiotic resistance. Although there is some concern regarding potential for autolysis and subsequent acquisition of resistance, the potential dilution effect would conceivably rule out such a mechanism. Additionally, the use of antibody to the cat gene product to demonstrate accumulation of Cat over time in the recipient cells deftly avoided issues concerning spontaneous antibiotic resistance. Although protein was shown to transfer, demonstration of the transfer of RNA and metabolites were not. Evidence of RNA or metabolite transfer would have provided additional support for the general hypothesis, including the potential rescue of auxotrophic mutants and bacterial adaptation. Although the authors suggest that transfer of mRNA may be occurring in addition to transfer of the gene products, experiments designed to address this question were not performed. Possibilities include utilizing donor cells that are sensitive to inhibition of protein synthesis with resistant recipient cells to express the fluorescent protein.

Of course, the critical question is whether the exchange of cytoplasmic material might include transfer of DNA. Similar to previous experiments documenting the transfer of non-hereditary material, co-cultivation of bacteria was shown to result in the exchange of antibiotic resistance. The frequency of transfer was shown to be at least 1000-fold less than that observed with conjugative transfer. This result is somewhat surprising since the number and frequency of connections may be thought to be more numerous, based on the EM images, than via conjugation which was performed using a 1:1 mixture of donor to recipient. Perhaps the specialized design of the conjugation system enhances the transport of DNA. Finally, and perhaps most importantly, HR-SEM clearly revealed the presence of nanotubes formed between different organisms including B. subtilis and Staphylococcus aureus as well as B. subtilis and E. coli to confirm the ubiquitous nature of this phenomenon.

\section{IMPACT ON BACTERIAL GENETICS}

Of obvious interest is the potential for exchange of non-hereditary and hereditary material between different bacterial species. The fact that bacteria share and exchange DNA is not a surprise.
Filaments or intracellular channels in cyanobacteria have been shown to mediate the movement of small molecules. A similar phenomenon has been previously observed in archaebacteria, where non-conjugative plasmids were shown to reciprocally traverse from one cell to another and cytoplasmic bridges were detected between cells (Rosenshine et al., 1989; Schleper et al., 1995). The appearance of antibiotic resistant variants of critical pathogens has long been documented, but the general nature of the mechanism had not been recognized. Recognition of a mechanism provides a means of exploring its contribution to the spread of antibiotic resistance and hopefully used to prevent the development of superbugs. By targeting the mechanism responsible for nanotube formation exchange of antibiotic resistance and introduction into species and genera for which they are not typically found may be approached. However, these results suggest that evaluation of alternative systems for DNA transfers should not be ignored. Gram-negative bacteria create extracellular membrane vesicles $(\mathrm{MVs}$ ) to trade information via packaged molecules (Lee et al., 2009; Schooling et al., 2009; Kitagawa et al., 2010; PradosRosales et al., 2011; Tashiro et al., 2011). Is it possible to exchange hereditary material using these pathways? Cell-to-cell exchange of information via signaling molecules belonging to low molecular weight autoinducers and signaling oligopeptides has been described for more than two decades.

There are at least six secretion systems characterized in bacteria that function to secret or inject proteins into the environment and in some cases directly into target cells to prepare them for infection (Durand et al., 2009; Fronzes et al., 2009). The type I, II, V, and VI transport systems appear to be designed for release of proteins into the neighboring environment to enhance survival, the best example being the hemolysin genes designed to rupture target cells. The type III and IV systems are of particular interest since they derive from bacterial systems for motility and conjugation and have been adapted in such as way as to inject proteins and DNA into the cell in order to prepare it for successful infection. The T4SS retains the ability to inject DNA into target cells as the progenitor conjugation system was designed to do. Whether this is used for exchange between bacteria has not been documented.

At this point there is little to go on concerning the precise purpose of these systems. Neighboring cells were shown to be connected by cytoplasmic tubes called "plasmodesmata" and the phenomenon was observed between disparate organisms including between Gram-positive organisms B. subtilis and S. aureus, and even between B. subtilis and the Gram-negative bacterium E. coli. Such broad reactivity has not been observed with classical conjugation systems. The fact that this phenomenon was not readily observed in liquid culture was interpreted as a sign of induction specifically under this growth condition. Although this cannot be ruled out at this point, alternative explanations include potential sensitivity to shearing forces when agitated in culture and indicates a propensity to detect such bridges when growing on solid surfaces or perhaps in biofilms. However, these results also suggest the potential for the presence of mixed populations of bacteria when grown on solid surfaces. The capacity of the progenitor organism to "sustain" more than one variant may enhance survival of the organism confronted with rapidly changing environmental 
conditions. The capacity for bacteria to anticipate such changes has been documented in so-called contingency loci, however, this rapid adaptation may simply reflect the ongoing accumulation of mutants that are maintained by cross-feeding by the progenitor organism.

The work of Dubey and Ben-Yehuda (2011) provides the basic premise for exploration of at least two critical aspects of bacterial genetics. The first, is the broad range sharing of antibiotic resistance and the second is the apparent capacity of bacteria to anticipate changes in its environment. It should not be assumed that nanotubes represent the only mechanism behind either of these two phenomena, but the potential for being key contributors is obvious, and should be explored further. However, even more importantly documentation of such a general mechanism suggests that other general phenomenon should be examined for their contribution to these important events. If as proposed, nanotube-mediated cytoplasmic sharing represents a key form of intercellular bacterial communication in nature that includes communication by immediate transfer of information capable

\section{REFERENCES}

Avery, O. T., Macleod, C. M., and Mccarty, M. (1944). Studies on the chemical nature of the substance inducing transformation of pneumococcal types: induction of transformation by a desoxyribonucleic acid fraction isolated from pneumococcus type III. J. Exp. Med. 79, 137-158.

Brock, T. D. (1990). The Emergence of Bacterial Genetics. Cold Spring Harbor, NY: Cold Spring Harbor Laboratory Press.

Dubey, G. P., and Ben-Yehuda, S. (2011). Intercellular nanotubes mediate bacterial communication. Cell 144, 590-600.

Durand, E., Verger, D., Rego, A. T., Chandran, V., Meng, G., Fronzes, R., and Waksman, G. (2009). Structural biology of bacterial secretion systems in gram-negative pathogens - potential for new drug targets. Infect. Disord. Drug Targets 9, 518-547.

Fronzes, R., Christie, P. J., and Waksman, G. (2009). The structural biology of type IV secretion systems. Nat. Rev. Microbiol. 7, 703-714.

Hayes, W. (1952a). Genetic recombination in Bact. coli K12; analysis of the stimulating effect of ultra-violet light. Nature 169, 1017-1018.

Hayes, W. (1952b). Recombination in Bact. coli $\mathrm{K}$ 12; unidirectional transfer of genetic material. Nature $169,118-119$.

Kitagawa, R., Takaya, A., Ohya, M., Mizunoe, Y., Takade, A., Yoshida, S., Isogai, E., and Yamamoto, T. (2010). Biogenesis of Salmonella enterica serovar typhimurium membrane vesicles provoked by induction of PagC. J. Bacteriol. 192, 5645-5656.

Lederberg, J. (1947). Gene recombination and linked segregations in Escherichia coli. Genetics 32, 505-525.

Lederberg, J. (1987). Genetic recombination in bacteria: a discovery account. Annu. Rev. Genet. 21, 23-46.

Lederberg, J., and Tatum, E. (1946). Novel genotypes in mixed cultures of biochemical mutants of bacteria. Cold Spring Harb. Symp. Quant. biol. 11, 113-114.

Lee, E. Y., Choi, D. Y., Kim, D. K., Kim, J. W., Park, J. O., Kim, S., Kim, S. H., Desiderio, D. M., Kim, Y. K., Kim, K. P., and Gho, Y. S. (2009). Gram-positive bacteria produce membrane vesicles: proteomics-based characterization of Staphylococcus aureus-derived membrane vesicles. Proteomics 9, 5425-5436.

Prados-Rosales, R., Baena, A., Martinez, L. R., Luque-Garcia, J., Kalscheuer, R., Veeraraghavan, U., Camara, C.,

of crossing inherent species barriers, than we may go along way toward preventing the appearance of superbugs.

\section{SUMMARY}

Despite this carefully performed study the fact that bacteria remain divided into discrete genera and species suggests that the capacity to stably exchange DNAs may be limited, and at least for now, the phenomenon should be carefully studied with emphasis on resolving the following observations.

(1) Is this mechanism responsible for the broad range genetic exchange responsible for the spread of antibiotic resistance?

(2) Is there a restriction on the size of the DNA that may be transferred?

(3) Does the exchange of cytoplasmic components play a role in bacterial adaptation?

(4) Does transfer occur under unusual growth conditions designed to provide protection?

(5) Is there a requirement for cell viability necessary for exchange?

Nosanchuk, J. D., Besra, G. S., Chen, B., Jimenez, J., GlatmanFreedman, A., Jacobs, W. R. Jr., Porcelli, S. A., and Casadevall, A. (2011). Mycobacteria release active membrane vesicles that modulate immune responses in a TLR2dependent manner in mice. J. Clin. Invest. 121, 1471-1483.

Rosenshine, I., Tchelet, R., and Mevarech, M. (1989). The mechanism of DNA transfer in the mating system of an archaebacterium. Science 245, 1387-1389.

Schleper, C., Holz, I., Janekovic, D., Murphy, J., and Zillig, W. (1995). A multicopy plasmid of the extremely thermophilic archaeon Sulfolobus effects its transfer to recipients by mating. J. Bacteriol. 177, 4417-4426.

Schooling, S. R., Hubley, A., and Beveridge, T. J. (2009). Interactions of DNA with biofilm-derived membrane vesicles. J. Bacteriol. 191, 4097-4102.

Tashiro, Y., Inagaki, A., Shimizu, M., Ichikawa, S., Takaya, N., NakajimaKambe, T., Uchiyama, H., and Nomura, N. (2011). Characterization of phospholipids in membrane vesicles derived from Pseudomonas aeruginosa. Biosci. Biotechnol. Biochem. 75, 605-607.

Tatum, E. L., and Lederberg, J. (1947). Gene recombination in the bacterium Escherichia coli. J. Bacteriol. 53, 673-684.

Tiberghien, F., and Loor, F. (1996). Ranking of P-glycoprotein substrates and inhibitors by a calcein-AM fluorometry screening assay. Anticancer Drugs 7, 568-578.

Conflict of Interest Statement: The author declares that the research was conducted in the absence of any commercial or financial relationships that could be construed as a potential conflict of interest.

Received: 18 April 2011; paper pending published: 09 June 2011; accepted: 16 August 2011; published online: 08 September 2011.

Citation: Ficht TA (2011) Bacterial exchange via nanotubes: lessons learned from the history of molecular biology. Front. Microbio. 2:179. doi: 10.3389/fmicb.2011.00179

This article was submitted to Frontiers in Evolutionary and Genomic Microbiology, a specialty of Frontiers in Microbiology. Copyright $\odot 2011$ Ficht. This is an openaccess article subject to a non-exclusive license between the authors and Frontiers Media SA, which permits use, distribution and reproduction in other forums, provided the original authors and source are credited and other Frontiers conditions are complied with. 